\title{
Évolution possible des débits d'étiage de la Meuse en France au cours du XXI ${ }^{\text {ème }}$ siècle
}

Possible evolution of French Meuse low flows during the XXI century

Didier François, Gilles Drogue et Fabien Commeaux

\section{(2) OpenEdition \\ 9 Journals}

\section{Édition électronique}

URL : http://journals.openedition.org/rge/3416

DOI : $10.4000 /$ rge.3416

ISSN : 2108-6478

\section{Éditeur}

Association des géographes de l'Est

\section{Édition imprimée}

Date de publication : 23 août 2011

ISSN : 0035-3213

Référence électronique

Didier François, Gilles Drogue et Fabien Commeaux, «Évolution possible des débits d'étiage de la Meuse en France au cours du XXIème siècle », Revue Géographique de l'Est [En ligne], vol. 51 / 3-4 | 2011 , mis en ligne le 28 août 2012, consulté le 08 septembre 2020. URL : http://journals.openedition.org/ rge/3416 ; DOI : https://doi.org/10.4000/rge.3416

Ce document a été généré automatiquement le 8 septembre 2020

Tous droits réservés 


\section{Évolution possible des débits d'étiage de la Meuse en France au cours du XXI ${ }^{\text {ème }}$ siècle}

Possible evolution of French Meuse low flows during the XXI century

Didier François, Gilles Drogue et Fabien Commeaux

\section{Introduction}

1 En 2009, l'EPAMA (Établissement Public d'Aménagement de la Meuse et de ses Affluents) a initié un projet concernant des solutions d'adaptation de la Meuse aux impacts des extrêmes hydrologiques liés au changement climatique. Ce projet a pris le nom d'AMICE pour : Adaptation of the Meuse to the Impacts of Climate Evolution ${ }^{1}$ et a été retenu par le programme européen INTERREG IVB ENO. Il regroupe 17 partenaires provenant des 4 pays contrôlant une partie du bassin versant de la Meuse (France, Belgique, Allemagne et Pays-Bas).

2 Nous présentons ici un des aspects d'AMICE relatif à l'estimation de l'évolution des débits d'étiage de la Meuse au cours du XXI ${ }^{\text {ème }}$ siècle (Drogue \& al., 2010). La méthode choisie consiste à alimenter un modèle hydrologique empirique par des données de scénarios climatiques (un scénario sec et un scénario sec modéré). Elle tient compte également de l'incertitude liée à l'estimation des débits par le modèle. La valeur du débit mensuel d'étiage (QMNA) de fréquence $1 / 5$ calculée sur 3 périodes trentenaires (1971-2000, 2021-2050 et 2071-2100) sert d'indicateur de cette évolution.

\section{Méthodologie}

3 La démarche mise en œuvre pour estimer les QMNA de la Meuse comporte 3 étapes (fig. 1) : 
Figure 1 : Méthodes mises en oeuvre

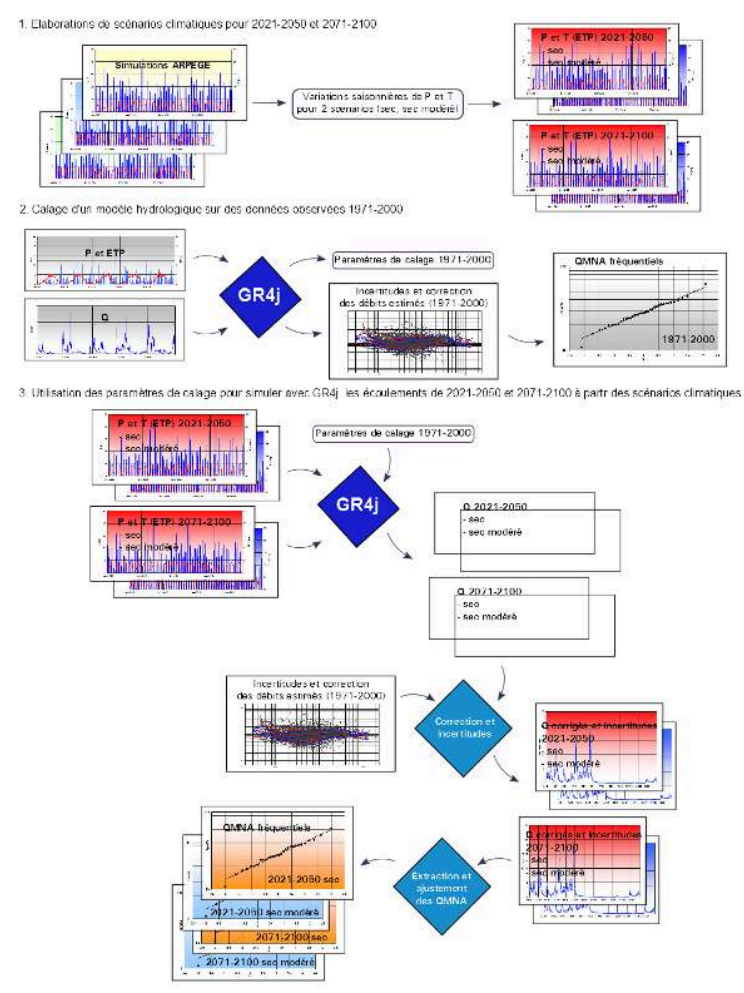

Dans un premier temps, nous nous sommes intéressés aux données issues de scénarios climatiques sur la base de simulations réalisées par le modèle ARPEGE-Climat v.4 de Météo- France. Le modèle numérique ARPEGE est un modèle de circulation générale global et spectral développé par Météo-France en collaboration avec le CEPMMT pour la prévision numérique du temps. La version climat d'ARPEGE, nommée ARPEGEClimat, a été développée dans les années 1990. Elle est dérivée du modèle de prévision opérationnelle à courte échéance. La grille d'ARPEGE peut être basculée pour changer la position du pôle et étirée pour augmenter la résolution dans une zone d'intérêt. Cette capacité de zoom a permis de développer des études régionales du climat avec ARPEGE-Climat notamment sur l'Europe (Déqué et Piedelievre, 1995). Dans la version climat d'ARPEGE, la résolution varie de $50 \mathrm{~km}$ au centre de la Méditerranée à $450 \mathrm{~km}$ dans le Pacifique Sud. Ceci assure une résolution d'au moins $60 \mathrm{~km}$ sur la France. La discrétisation verticale se fait sur 31 couches qui suivent le relief avec des tranches d'altitude variables. Le pas de temps de calcul est de $30 \mathrm{mn}$. Nous avons caractérisé le climat actuel (1971-2000²) à partir des données provenant de E-OBS 2.0. Cette base de données hébergée sur le site du "European Climate Assessment \& Dataset" regroupe des températures de l'air et des précipitations journalières spatialisées selon une grille de résolution de $0.25^{\circ}$ (environ $17 \mathrm{~km}$ en longitude et $27 \mathrm{~km}$ en latitude dans la zone du bassin de la Meuse) sur la période 1950-2008 (Haylock \& al., 2008). Les valeurs moyennes de températures et de précipitations aux stations hydrométriques résultent d'une spatialisation par des polygones de Thiessen. Les scénarios climatiques utilisés pour cette étude procèdent d'une estimation par Météo-France des tendances saisonnières pour les périodes 2021-2050 et 2071-2100 par rapport au climat actuel (méthode des anomalies). Ces tendances ont été appliquées globalement au bassin de la Meuse sans tenir compte de nuances locales qui apparaissent dans les données d'échelle fine (Commeaux et al., 2010). Elles concernent 2 scénarios d'émission de gaz à 
effet de serre siglés A2 et A1B appliqués au modèle ARPEGE Climat v.4. L'approche adoptée dans le cadre de cette analyse n'est donc pas ensembliste. Elle se fonde sur les simulations d'un seul modèle de circulation générale et ne prend en compte que les deux scénarios d'émission A1B et A2 produits dans le cadre des expériences numériques d'intercomparaison du 3e rapport d'avancement du GIEC paru en 2001. L'incertitude réflexive (Pagé et Terray, 2010) est donc prise en compte, mais de façon limitée. L'objectif est de fournir une première indication du signe et de l'intervalle de variation de la tendance affectant les débits d'étiage pour deux futurs scénarios climatiques répondant à deux trajectoires sociétales équiprobables: i) une trajectoire " médiane " (scénario A1B) tablant sur une forte croissance économique qui suppose des échanges mondiaux importants, ii) une trajectoire « tendancielle » (scénario A2). Ce dernier table sur une croissance économique qui met l'accent sur les échanges régionaux. Le scénario A2 est le plus pessimiste des scénarios utilisés et simule la situation qu'il adviendrait si aucune mesure n'était prise pour restreindre les émissions de gaz à effet de serre. Dans ce scénario la concentration en $\mathrm{CO} 2$ en 2100 est de 840 ppm contre 770 ppm pour le scénario A1B. Par souci de simplification nous désignons respectivement A2 et A1B, scénario "sec modéré" et scénario "sec". Pour obtenir des séries de données représentatives des périodes 2021-2050 et 2071-2100, les tendances saisonnières de chaque scénario exprimées en pourcentage pour la pluie et en écart absolu pour les températures de l'air ont été imputées aux données du climat actuel issues de la climatologie E-OBS. Les valeurs d'évapotranspiration potentielle proviennent de l'application de la formule à base thermique de Oudin (Oudin \& al., 2005).

5 La deuxième phase a consisté à caler un modèle hydrologique pluie-débit journalier avec les données observées de la période 1971-2000. Nous avons utilisé le modèle GR4j (Perrin et al., 2003) car il est abondamment documenté3, il nécessite peu de paramètres et a déjà montré de bonnes performances (en comparaison avec d'autres modèles) sur la région d'étude. Son architecture est présentée sur la figure $n^{\circ} 2$. Pour son calage, nous avons utilisé comme fonction- objectif le critère de Nash-Sutcliffe calculé sur le logarithme des débits. Pour les 7 stations étudiées, les valeurs du critère de NashSutcliffe s'échelonnent de 77 à $90 \%$ et correspondent à des niveaux d'efficience satisfaisants pour simuler des QMNA. Les sorties du modèle pluie-débit font ensuite l'objet d'une correction et d'une estimation de leur incertitude selon une méthode empirique développée par Berthier (2005). Pour chaque classe de débits journaliers (d'amplitude exponentielle), on calcule le rapport médian débit journalier observé / débit journalier simulé (quantile $50 \%$ période de référence). Celui-ci sert de "coefficient correcteur" (par classe de débit) et permet d'améliorer la qualité des simulations (par exemple la correction du biais sur les faibles valeurs à Chalaines, fig. 3). Afin de s'assurer de la représentativité des résultats, les quantiles d'incertitude figurant sur le graphique concernent la période de calage (15 premières années, en bleu) et la période de référence ( 30 ans en rouge). Les paramètres de calage du modèle et les intervalles d'incertitudes déterminés ici pour la période de référence 1971-2000 serviront par la suite pour les simulations résultant des scénarios climatiques 2021-2050 et 2071-2100.

6 La troisième et dernière phase reprend les résultats et méthodes des deux premières. Les données des scénarios climatiques alimentent GR4j, les débits ainsi simulés passent l'opération de correction et au final, on extrait les valeurs de QMNA. Celles-ci sont enfin 
ajustées à une loi log-normale pour définir le quantile quinquennal sec (seuil fréquemment utilisé en France pour caractériser un étiage sévère).

Figure 2 : Le modèle GR4j

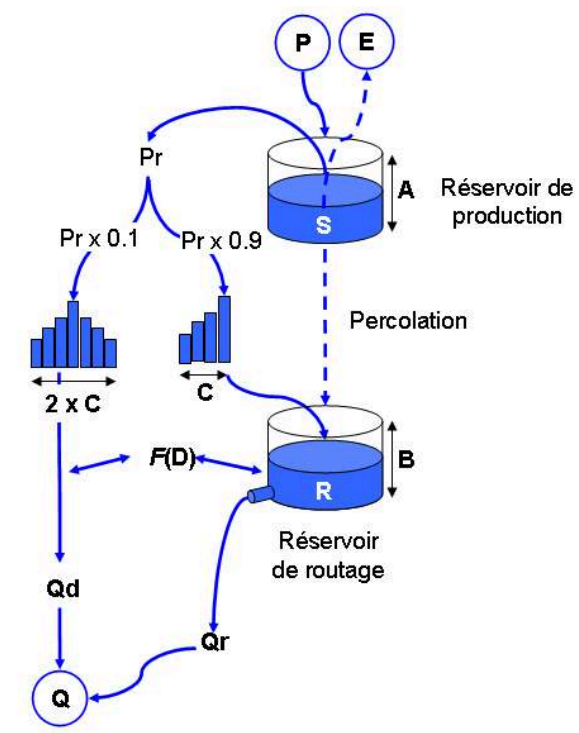

Le modèle GR4j (Perrin et al., 2003)

- 4 paramètres

- A : capacité maximale du réservoir de production (en $\mathrm{mm}$ )

- B : capacité maximale du réservoir de routage (en $\mathrm{mm}$ )

- C : durée de l'hydrogramme unitaire (en jours)

- D : échange inter-bassin (en mm)

- données d'entrée : précipitations et ETP journalières (mm/j)

- données produites : écoulements moyens journaliers $(\mathrm{mm} / \mathrm{j})$

Figure 3 : Correction et incertitudes des débits simulés - La Meuse à Chalaines
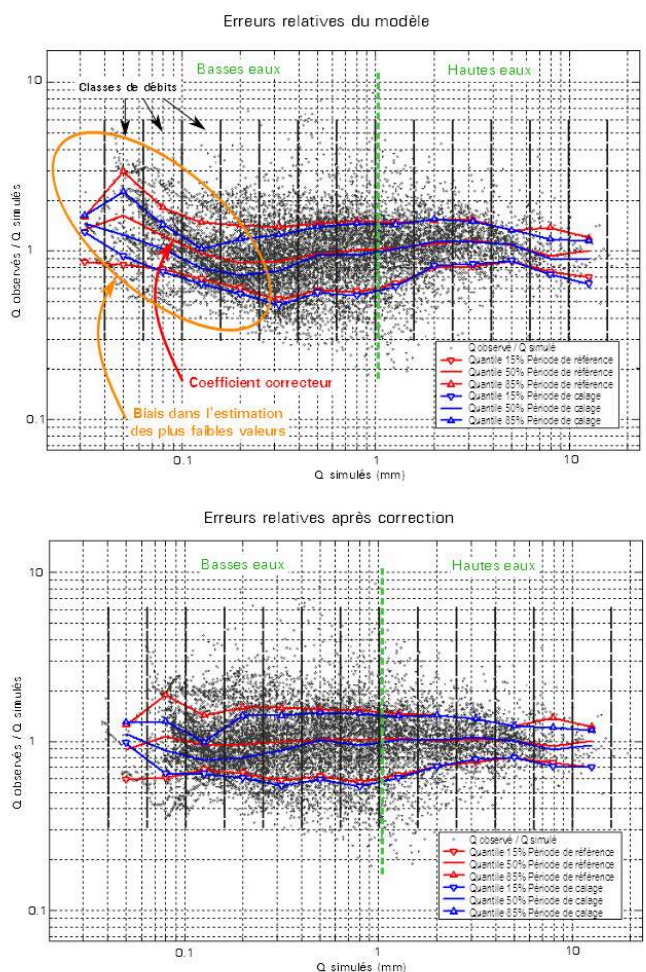


\section{Bassins étudiés}

$7 \quad$ Les bassins versants étudiés se situent principalement en France (fig. 4). Ils sont drainés par la Meuse et ses affluents. Celle-ci prend sa source dans la région du Bassigny. Jusqu'à la station de Goncourt, elle draine essentiellement des terrains imperméables marneux ou argileux. En aval jusqu'à la station de Stenay, le fleuve entaille successivement les plateaux calcaires du Dogger puis le plateau de l'Oxfordien. Les premiers concentrent une densité relativement importante de phénomènes karstiques qui peuvent être à l'origine de pertes (en amont de Neufchateau) et de circulations souterraines vers des bassins contigus (la Moselle ou la Marne). Ces échanges s'effectuent dans la plupart des cas aux dépends du bassin de la Meuse. En aval de Stenay, la Chiers, principal affluent de la Meuse (qui apparait particulièrement démembrée), draine en rive gauche les terrains du Dogger et en rive droite les grès d'Ardenne-Luxembourg qui assurent un soutien important en période d'étiage. En aval de Charleville-Mézières, la Meuse entaille le massif ardennais. Celui-ci ne dispose de ressource aquifère que très localement. Par contre il constitue le pôle pluviométrique du bassin avec ponctuellement plus de $1300 \mathrm{~mm}$ /an. Dans la partie amont du bassin la pluviométrie demeure très homogène avec des hauteurs annuelles comprises entre 800 et $900 \mathrm{~mm} / \mathrm{an}$.

8 Nous avons sélectionné 7 stations hydrométriques présentant des chroniques de débits relativement longues. La plus récente a débuté en 1973 (Chalaines) et toutes ces stations fonctionnent encore actuellement (Tableau 1). Elles sont toutes gérées par la DREAL Lorraine et leurs données proviennent de la Banque Hydro. Elles se répartissent assez régulièrement le long du cours de la Meuse avec des bassins versants relativement grands compris entre 364 et $10120 \mathrm{~km}^{2}$ (fig. 4). 
Figure 4 : Domaine d'étude

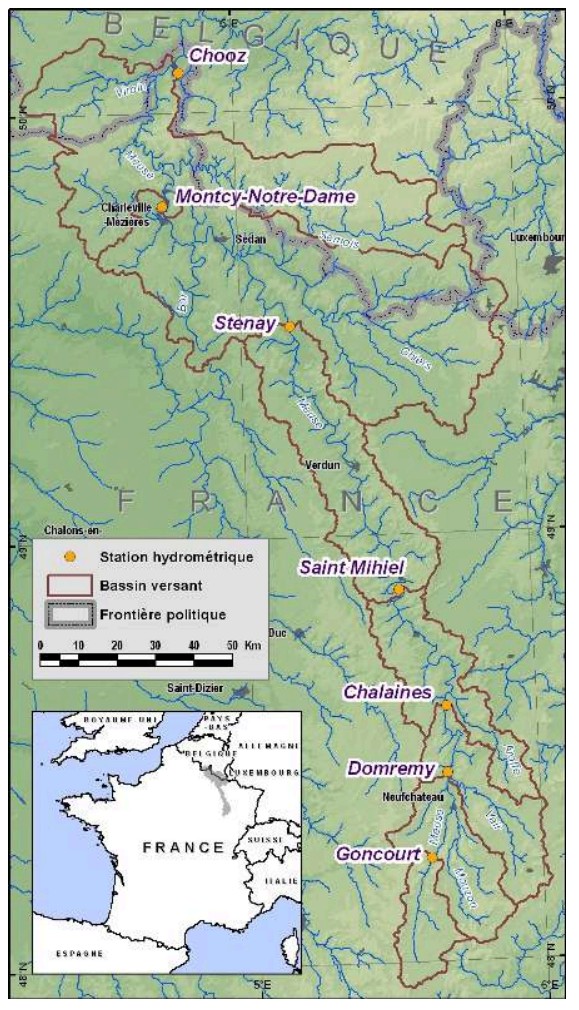

Tableau 1 : Stations hydrométriques étudiées

\begin{tabular}{|c|l|c|c|}
\hline Code & \multicolumn{1}{|c|}{ Nom } & $\begin{array}{c}\mathbf{1}^{\text {ére }} \text { année de } \\
\text { fonctionnement }\end{array}$ & $\begin{array}{c}\text { Surface de bassin } \\
\text { versant }\left(\mathbf{k m}^{\mathbf{2}}\right)\end{array}$ \\
\hline B0220010 & Goncourt & 1971 & 364 \\
\hline B1150010 & Domrémy-la-Pucelle & 1969 & 1031 \\
\hline B1340010 & Vaucouleurs [Chalaines] & 1973 & 1717 \\
\hline B2220010 & Saint-Mihiel & 1968 & 2540 \\
\hline B3150020 & Stenay & 1963 & 3904 \\
\hline B5600010 & Montcy-Notre-Dame & 1972 & 7724 \\
\hline B7200010 & Chooz & 1953 & 10120 \\
\hline
\end{tabular}

\section{Résultats}

\section{A. Scénarios climatiques}

Les sorties d'ARPEGE Climat pour les 2 scénarios indiquent une augmentation des températures pour toutes les saisons. Comparativement à 1971-2000 cette hausse se situe entre $+1.2^{\circ} \mathrm{C}$ et $+2.1^{\circ} \mathrm{C}$ pour $2021-2050$ et $+2.6^{\circ} \mathrm{C}$ à $+5.6^{\circ} \mathrm{C}$ pour 2071-2100 (Tableau 2). Pour la modélisation hydrologique, l'ETP répercute cette évolution car son estimation s'appuie uniquement sur les données de température de l'air.

10 A cette élévation de la température de l'air s'ajoute une diminution globale des précipitations saisonnières pouvant aller jusqu'à -39\% (été 2071-2100, Tableau 2). Le scénario sec modéré envisage juste une petite augmentation $(+4 \%)$ des précipitations 
printanières sur la période 2021-2050. L'écart entre les 2 scénarios s'avère peu important hormis pour les précipitations hivernales 2071-2100.

Tableau 2 : Scénarios climatiques, évolution des cumuls de précipitations et des températures moyennes

\begin{tabular}{|c|c|c|c|c|c|c|c|}
\hline Variable & Période & Scénario & Hiver & Printemps & Été & Automne & Année \\
\hline \multirow{4}{*}{$\begin{array}{l}\text { Ecart absolu des } \\
\text { températures } \\
\text { moyennes }\left(\mathrm{en}^{\circ} \mathrm{c} \text { ) }\right.\end{array}$} & \multirow{2}{*}{$2021-2050$} & Sec modéré & +1.3 & $\begin{array}{l}1.6 \\
\end{array}$ & +2.1 & $\begin{array}{r}+1.5 \\
\end{array}$ & +1.6 \\
\hline & & $\mathrm{Sec}$ & +1.4 & +1.2 & +1.7 & +1.3 & +1.4 \\
\hline & \multirow{2}{*}{$2071-2100$} & Sec modéré & +3.4 & +3.2 & +5.6 & +4.2 & +4.1 \\
\hline & & $\mathrm{Sec}$ & +2.6 & +2.7 & +4.5 & +3.3 & +3.3 \\
\hline \multirow{4}{*}{$\begin{array}{l}\text { Variation des cumuls } \\
\text { de précipitations }\end{array}$} & \multirow{2}{*}{$2021-2050$} & Sec modéré & $-7 \%$ & $4 \%$ & $-11 \%$ & $-5 \%$ & $-5 \%$ \\
\hline & & Sec & $-9 \%$ & $-1 \%$ & $-9 \%$ & $-13 \%$ & $-8 \%$ \\
\hline & \multirow{2}{*}{$2071-2100$} & Sec modéré & $-9 \%$ & $-11 \%$ & $-29 \%$ & $-22 \%$ & $-18 \%$ \\
\hline & & Sec & $-25 \%$ & $-11 \%$ & $-39 \%$ & $-22 \%$ & $-24 \%$ \\
\hline
\end{tabular}

\section{B. Simulation des débits d'étiage}

11 Les principaux résultats sont présentés sous la forme de profils hydrologiques qui mettent en relation l'évolution des débits d'amont en aval avec la surface du bassin versant drainé.

- Période de référence (1971-2000)

12 Le profil de la Meuse permet de juger la capacité du modèle hydrologique à simuler les débits mensuels d'étiage. Pour Goncourt (station la plus en amont) le modèle peine à produire des simulations d'étiage comparables aux valeurs observées (fig 5). Avec les valeurs simulées et corrigées, on atteint à peine la borne supérieure de l'intervalle de confiance statistique (90\%) de l'ajustement des QMNA. Il s'agit du plus petit bassin versant $\left(364 \mathrm{~km}^{2}\right)$ pour lequel les précipitations moyennes issues de la climatologie EOBS sont peut-être sur-estimées. 
Figure 5 : Profil hydrologique de la Meuse française - Débits mensuels d'étiage quinquennal sec Période 1971-2000

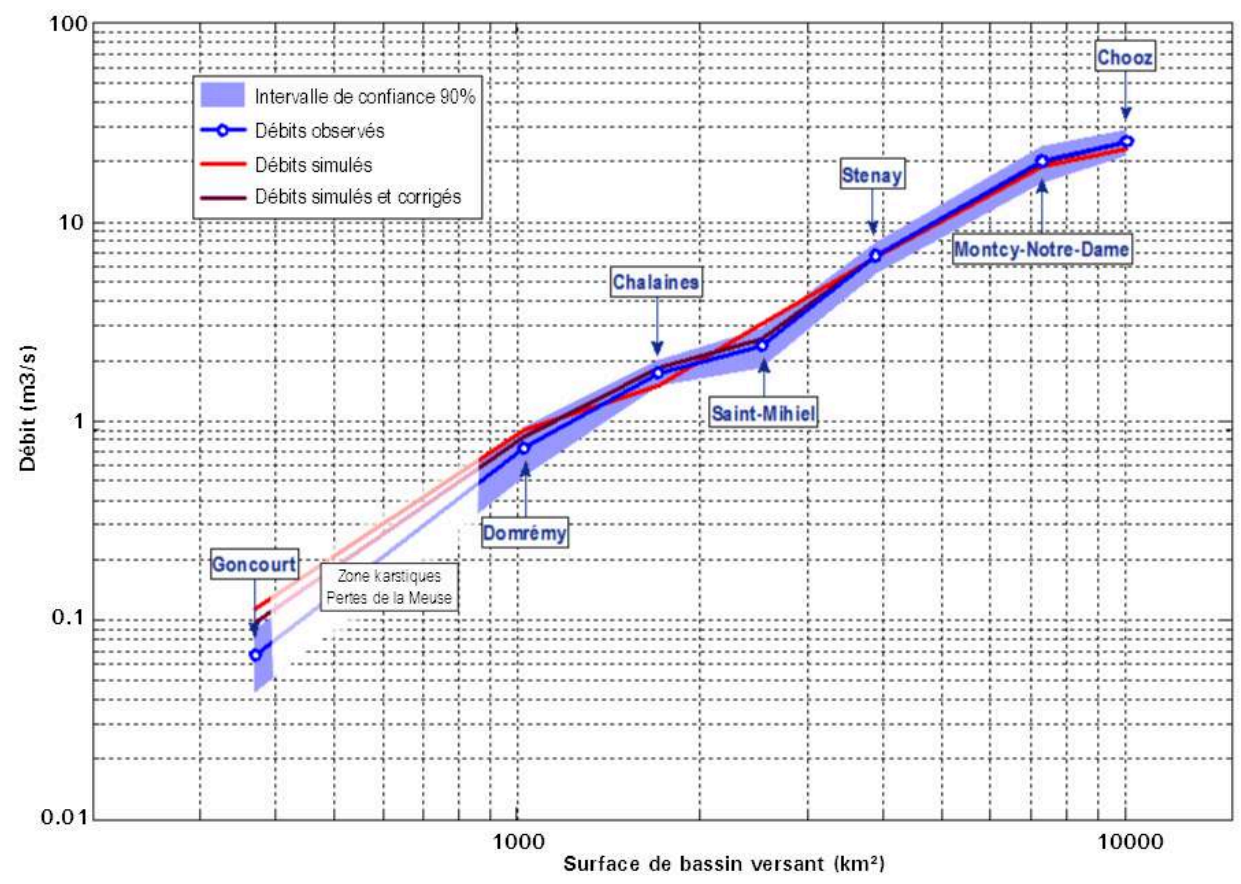

En revanche, pour les stations aval avec des bassins versants supérieurs à $1000 \mathrm{~km}^{2}$, le modèle présente des QMNA simulés et corrigés proches des QMNA observés (profils confondus à partir de Stenay). La procédure de correction exposée plus haut (Berthier, 2005) renforce parfois très avantageusement la qualité des simulations comme par exemple à Saint-Mihiel.

- QMNA scénario sec et QMNA scénario sec modéré

14 Si les scénarios climatiques sec et sec modéré présentent, somme toute, assez peu d'écarts (cf. § 3.1), les QMNA produits par GR4j à partir de ces scénarios se différencient encore moins (Tableau 3). Quelle que soit l'échéance envisagée (2021-2050 ou 2071-2100), nous pouvons considérer qu'il n'existe qu'une seule hypothèse de travail, les deux scénarios aboutissant à des résultats similaires. L'écart significatif constaté pour les précipitations hivernales ne se répercute pas sur les débits d'étiage simulés.

Nous avons choisi d'adopter comme référence les QMNA 1/5 simulés et corrigés pour calculer les écarts avec les QMNA des périodes 2021-2050 et 2071-2100 car ces derniers résultent des mêmes procédures (modélisation et correction). S'il existe un biais comme à Goncourt, on peut concevoir que les valeurs de QMNA projetées pour nos périodes-objectifs égalent le QMNA observé plus les écarts présentés dans le tableau 3. 
Tableau 3 : Débits mensuels d'étiage quinquennaux sec (QMNA 1/5)

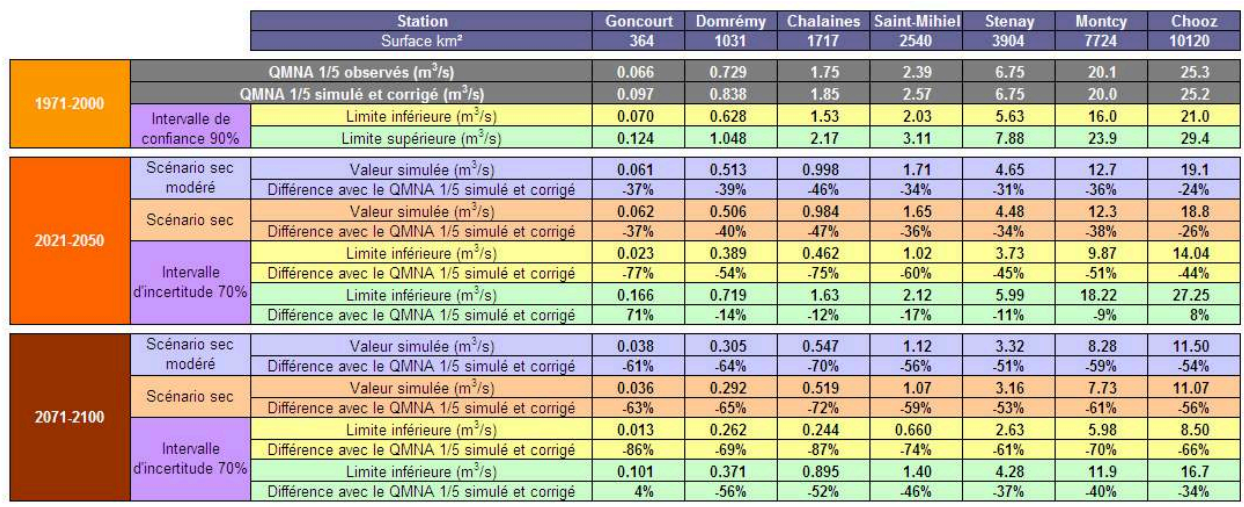

- Période 2021-2050

Dans la partie amont du bassin, en raison des incertitudes du modèle, on ne peut pas clairement estimer une décroissance significative du QMNA 1/5 2021-2050 par rapport à la période 1971-2000 (fig. 6). Par contre de Chalaines à Montcy-Notre-Dame, cette baisse apparaît plus marquée avec des valeurs relatives comprises entre -28 et $-44 \%$ (Tableau 3). Le profil hydrologique 1971-2000 ne chevauche pas le rail d'incertitude à 70 $\%$ des QMNA 1/5 simulés de la période 2021-2050. Par contre à l'extrémité aval du bassin, l'écart entre les QMNA des 2 périodes s'atténue (-26\% à $-25 \%)$ et le QMNA 1/5 2021-2050 dépasse la borne inférieure de l'intervalle de confiance à 90\% du QMNA 1/5 1971-2000.

Figure 6 : Profil hydrologique de la Meuse française - Débits mensuels d'étiage quinquennal sec Période 1971-2000 vs 2021-2050

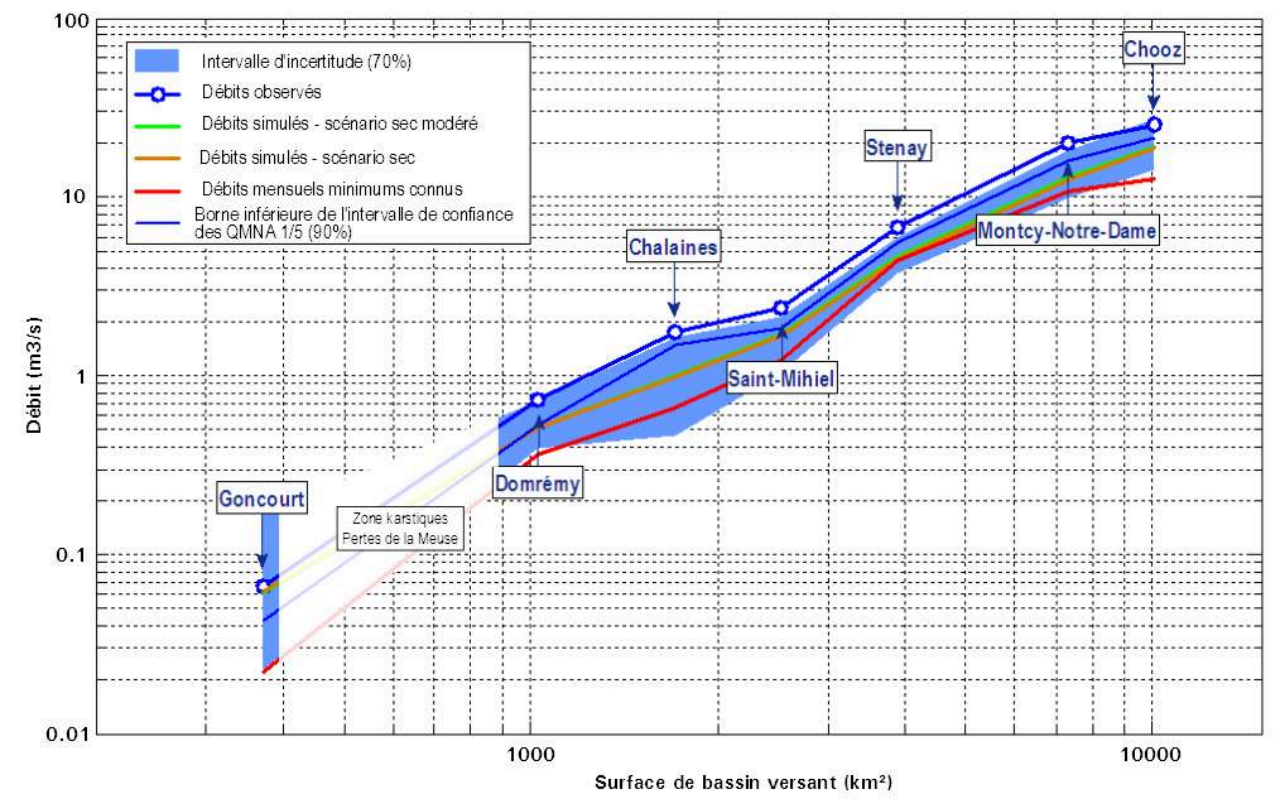

17 L'indication la plus spectaculaire concerne les stations de Stenay et Montcy où le QMNA 1/5 2021-2050 équivaudrait au débit mensuel d'étiage minimum connu sur la période de référence 1971-2000. Cet étiage correspond à l'année 1976 habituellement perçue comme particulièrement sévère voire "catastrophique". En fait, en suivant notre 
hypothèse de travail et pour ces 2 stations, d'ici le milieu du XXI ème siècle, les niveaux d'étiage de 1976 seraient sous-passés en moyenne tous les 5 ans.

- Période 2071-2100

Pour la fin du XXI ${ }^{\text {ème }}$ siècle, l'écart des QMNA 1/5 avec ceux de la période 1971-2000 s'amplifie (fig. 7). Il n'y a qu'à Goncourt où le rail d'incertitude dépasse encore la valeur du climat actuel. Partout ailleurs le profil de la période 2071-2100 apparaît très proche du débit minimum journalier connu sur la période de référence 1971-2000. En d'autres termes pour situer une valeur qui statistiquement n'est pas rarissime (le QMNA 1/5), nous avons dû nous référer au minimum absolu de la période 1971-2000. D'ici à la fin du siècle, les valeurs de QMNA 1/5 devraient globalement diminuer de moitié.

Figure 7 : Profil hydrologique de la Meuse française - Débits mensuels d'étiage quinquennal sec Période 1971-2000 vs 2071-2100

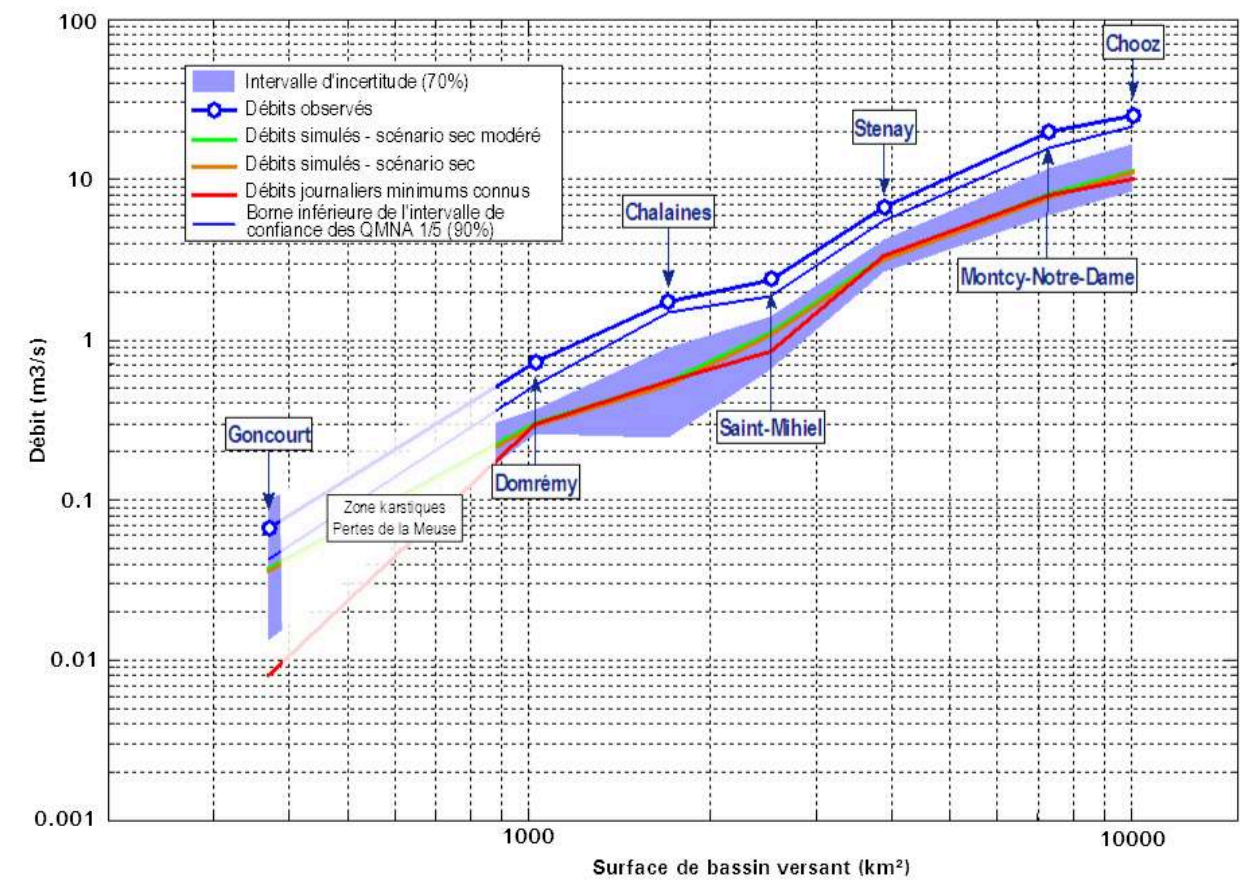

- Proportion de jours inférieurs au QMNA 1/5 1971-2000

Actuellement on se situe en moyenne 5\% (env. 18 jours/an) du temps sous le seuil du QMNA 1/5 (seuil de crise, fig. 8). On sous-passerait ce même seuil entre 6 (env. 22 jours/ an) et $22 \%$ (env. 80 jours/an) du temps en 2021-2050 et entre 15 (env. 55 jours/an) et 52\% (env. 200 jours/an) en 2071-2100. Notre définition des seuils de crise relatifs aux étiages se voient ainsi remis en cause et corrélativement certains usages de l'eau. 
Figure 8 : Proportion de débits journaliers inférieurs au QMNA 1/5 (intervalle d'incertitude de $70 \%$ inclus)

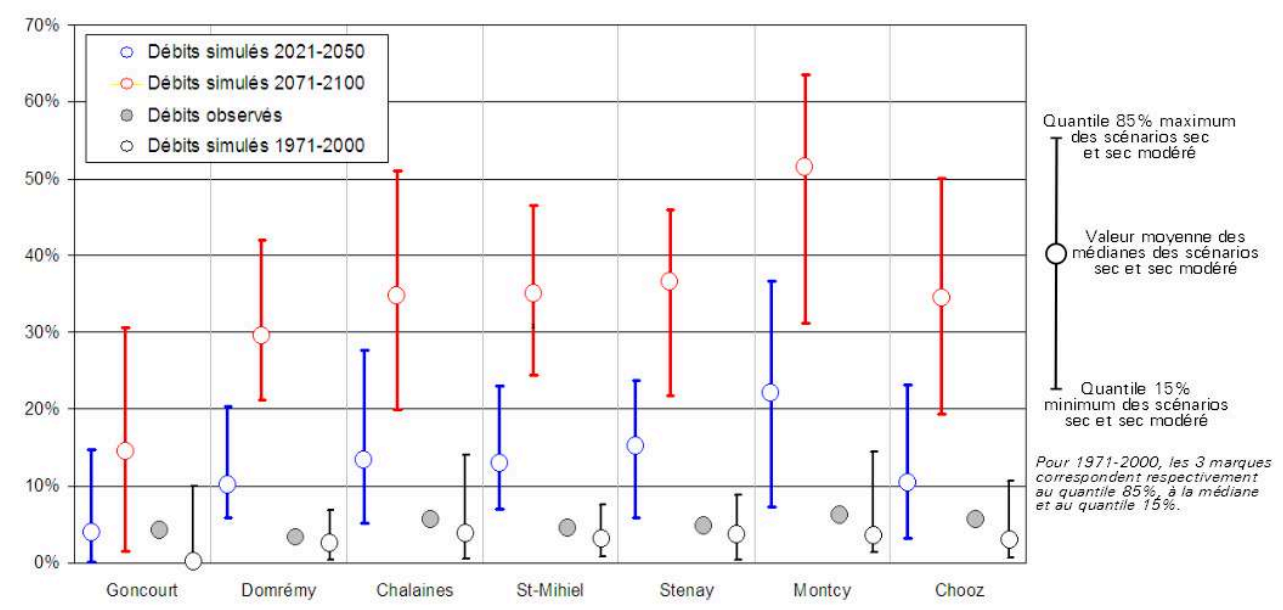

\section{Discussion}

A l'évidence, ce travail n'a pas comme ambition de représenter une prévision des débits d'étiage de la Meuse pour le XXI ${ }^{\text {ème }}$ siècle. Il s'agit davantage d'un "exercice de style" où la contrainte est de produire des débits d'étiage à partir de scénarios climatiques hypothétiques et équiprobables. Nous ne disposons d'aucun moyen pour valider nos simulations, les seules garanties que nous pouvons proposer concernent la qualité de la simulation climatique de référence et des débits simulés sur une période connue. La crédibilité des scénarios climatiques demeure prépondérante, pour que les débits d'étiage "possibles" proposés ici soient considérés comme des valeurs "probables". Ainsi un certain nombre d'hypothèses fortes ont jalonné notre démarche.

Pour la première phase, l'élaboration des scénarios climatiques résultant d'une modification globale des abats de précipitations journaliers par saison et de la température de l'air selon la même méthode peut paraître simpliste. Nous n'avons pas envisagé la possibilité d'apparition plus fréquente de longs épisodes secs aggravant les situations d'étiage. Ces épisodes peuvent également devenir plus durables.

Les simulations pour le XXI ${ }^{\text {ème }}$ siècle proviennent d'un modèle hydrologique calé sur la période 1971-2000. Ce calage nous semble robuste pour notre période de référence avec l'emploi d'une procédure de calage/contrôle. D'autre part nous avons testé différentes périodes de calage (années sèches, années humides) et constaté une faible sensibilité des paramètres. Mais dans le cadre non-stationnaire de l'évolution du climat que nous étudions, les paramètres imposés au modèle hydrologique pour 2021-2050 et 2071-2100 ne correspondent peut-être pas aux paramètres optimaux pour estimer les écoulements de ces périodes. Enfin les rails d'incertitude et la correction des débits simulés par le modèle hydrologique résultent également de calculs effectués sur notre période de référence. Leur transposition au XXI ${ }^{\text {ème }}$ siècle constitue un autre postulat d'autant plus téméraire que la distribution des débits simulés en fonction de leurs erreurs relatives (fig. 3) sera immanquablement différente. Une classe de débit peu représentée dans le climat actuel (comme les étiages les plus sévères) verra son effectif augmenter durant les périodes-objectifs (2021-2050 et 2071-2100). 
réserves s'appliquent à la plupart des travaux prospectifs. Notre démarche est surtout pragmatique et a pour souci de montrer l'incertitude parfois importante liée à des débits simulés malgré des critères d'ajustement du modèle pluie-débit satisfaisants. Elle montre aussi la nécessité de recourir à une démarche ensembliste permettant de probabiliser les futurs climatiques utilisés pour forcer les modèles d'impact en hydrologie.

\section{Conclusion}

24 enelle du bassin de la Meuse, l'évolution des QMNA 1/5 à partir d'un modèle hydrologique et en fonction des changements climatiques liés aux émissions additionnelles de gaz à effet de serre d'origine anthropique, n'apparait pas toujours décelable. Pour le petit bassin versant de la Meuse à Goncourt l'incertitude des estimations issues du modèle pluie-débit ne permet pas de démontrer une diminution significative même pour la période 2071-2100.

En revanche aux autres stations, si pour notre première période-objectif (2021-2050) la baisse des QMNA 1/5 peut sembler modérée, pour la seconde (2071-2100) la chute serait spectaculaire : le QMNA 1/5 se rapprocherait du débit journalier minimum connu sur la période 1971-2000.

Les partenaires du projet AMICE vont proposer une stratégie d'adaptation sous-tendue par cette évolution "possible" des ressources en eau superficielle d'ici la fin du XXIe siècle.

Du côté des géographes hydrologues et des hydrologues "globaux », ce type de travail prospectif lié aux évolutions du climat fait l'objet de nombreuses publications et ouvre des pistes de recherche particulièrement intéressantes (Brigode, 2010).

\section{BIBLIOGRAPHIE}

Berthier, C.H., (2005). Quantification des incertitudes des débits calculés par un modèle pluiedébit empirique. Mémoire Master 2 Hydrologie Hydrogéologie, Université Paris-Sud 11, Orsay, 55 p.

Boé J., 2007, Changement global et cycle hydrologique: Une étude de régionalisation sur la France, Thèse, Université Toulouse III - Paul Sabatier.

Brigode P., 2010, Modélisation hydrologique en contexte de changement climatique : Incertitudes liées aux paramètres de modèles conceptuels pluie-débit, Master 2 Sciences de l'Univers, Environnement, Ecologie. Parcours Hydrologie-Hydrogéologie, Université Pierre et Marie Curie, École des Mines de Paris \& École Nationale du Génie Rural des Eaux et des Forêts.

Commeaux F., Drogue G., François D., 2010, « Analyse prospective des débits mensuels d'étiage de la Meuse française à l'aide d'un modèle hydrologique : sensibilité aux méthodes de descente 
d'échelle spatiale et aux scénarios d'émission ", Bulletin de l'Association des Géographes Français, vol. 87, no2, pp. 178-193

Déqué M., Piedelievre J.-P., 1995, “High-Resolution climate simulation over Europe”, Climate Dynamics, 11:321-339.

Drogue G., Fournier M., Bauwens A., Buiteveld H., Commeaux F., Degré A., De Keizer O., Detrembleur S., Dewals B., François D., Guilmin E., Hausmann B., Hissel F., Huber N., Lebaut S., Losson B., Kufeld M., Nacken H., Pirotton M., Pontégnie D., Sohier C., Vanneuville W., 2010, Analysis of climate change, high - flows and low-flows scenarios on the Meuse basin, Projet AMICE, WP1 Action 3, http://www.amice-project.eu/docs/pa1_pr4_1286209557_WP1_1_Report.pdf

Haylock M.R., Hofstra N., Klein Tank A.M.G., Klok E.J., Jones P.D., New M., 2008, “A European daily high-resolution gridded dataset of surface temperature and precipitation", J. Geophys. Res (Atmospheres), 113, D20119, doi:10.1029/2008JD10201

Lang C., Freyermuth A., Gille E., François D., 2006, « PRESAGES (PREvisions et Simulations pour l'Annonce et la Gestion des Etiages Sévères) : Un outil pour évaluer et prévoir les étiages », Géocarrefour, Vol. 81, n¹, pp 15-23.

Oudin L., Michel C., Anctil, F., 2005, “Which potential evapotranspiration input for a rainfallrunoff model? Part 1 - Can rainfall-runoff models effectively handle detailed potential evapotranspiration inputs?", Journal of Hydrology, 303, 275-289.

Pagé C., Terray L., Boé J., 2008, Projections climatiques à échelle fine sur la France pour le 21ème siècle: Les scénarii SCRATCH08, Technical Report TR/CMGC/08/64, CERFACS.

Pagé C., Terray L., Boé J., 2009, dsclim: A software package to downscale climate scenarios at regional scale using a weather-typing based statistical methodology, Technical Report TR/CMGC/09/21, CERFACS.

Pagé C., Terray L., 2010, Nouvelles projections climatiques à échelle fine sur la France pour le 21ème siècle : les scénarii SCRATCH2010, Technical Report TR/CMGC/10/58, SUC au CERFACS, URA CERFACS/ CNRS No1875, Toulouse, France.

Perrin C., Michel C., Andréassian V., 2003, „Improvement of a parsimonious model for streamflow simulation", Journal of Hydrology, 279: 275-289.

\section{NOTES}

1. http://www.amice-project.eu

2. Nous avons choisi cette période car nous disposions déjà d'un référentiel statistique sur les débits d'étiage de la Meuse réalisé dans le cadre de l'étude PRESAGES (Lang et al., 2006)

3. http://www.cemagref.fr/webgr/

\section{RÉSUMÉS}

Cette étude a été menée dans le cadre du projet européen INTERREG IVB AMICE (Adaptation of the Meuse to the Impacts of Climate Evolution). Le principal objectif est de tester la réactivité des 
écoulements de la Meuse française en fonction i) des sorties d'un modèle climatique et ii) des incertitudes d'un modèle pluie-débit. Pour cela, une méthode de génération de scénarios climatiques (méthode des anomalies) a été appliquée aux simulations du modèle climatique ARPEGE-Climat forcé par 2 scénarios d'émission de gaz à effet de serre du GIEC. Ces scénarios climatiques ont ensuite alimenté un modèle hydrologique pluie-débit calé sur la période de référence 1971-2000. En réponse à ce forçage climatique, les valeurs des débits mensuels d'étiage simulées par le modèle pluie-débit ne représenteront plus que la moitié de leur valeur actuelle.

This study has been conducted in the framework of the European INTERREG IVB AMICE (Adaptation of the Meuse to the Impacts of Climate Evolution) project. The main objective is to show the sensitivity of the hydrological response of the French Meuse river to i) the outputs of a Limited-Area climate Model and ii) the uncertainty of the rainfall-runoff model. To achieve this goal, a method of climate scenario generation (the delta change approach) has been applied to the simulations of the ARPEGE-Climat model forced with 2 IPCC emission scenarios. The latter were further used as inputs of a rainfall-runoff model calibrated on the 1971-2000 reference period. In response to this climate forcing, low flow values for a 5 year return period will be halve of their present amounts.

\section{INDEX}

Keywords : climatic change scenarios, low flows, rainfall-runoff model, uncertainty

Mots-clés : étiage, incertitude, modèle pluie-débit, projections climatiques

\section{AUTEURS}

\section{DIDIER FRANÇOIS}

Université Paul Verlaine-METZ, CEGUM, UFR Sciences Humaines et Arts, Ile du Saulcy, BP 30309, 57006 METZ Cedex 1, didier.francois@univ-metz.fr

\section{GILLES DROGUE}

Université Paul Verlaine-METZ, CEGUM, UFR Sciences Humaines et Arts, Ile du Saulcy, BP 30309, 57006 METZ Cedex 1, drogue@univ-metz.fr

\section{FABIEN COMMEAUX}

Chargé de mission au Département de Seine-et-Marne, fabien.commeaux@gmail.com 